\title{
Controlling Hypertension: We Have the Tools-We Just Need to Use Them
}

\author{
David J. Hyman, MD, MPH and Valory Pavlik, PhD
}

Baylor College of Medicine, Houston, TX, USA.

$\mathrm{J}$ Gen Intern Med 32(8):860-2

DOI: $10.1007 / \mathrm{s} 11606-017-4060-8$

(c) Society of General Internal Medicine 2017

B lood pressure control among treated hypertensives has been improving in recent decades. Poor control in the past was largely attributed to "clinical inertia"-i.e., clinicians not increasing or adding antihypertensives in the face of uncontrolled blood pressure (BP), with nonadherence accounting for only $13 \%$ of the poor control. ${ }^{1}$ Physicians are now much more likely to add a second or third drug to reach the BP treatment target of less than 140/90 mmHg. Although BP control is usually achieved with three-drug therapy, some studies have suggested that many patients on three drugs, including a diuretic, were not controlled, and therefore potentially met criteria for resistant hypertension. Several studies published between 2003 and 2008 found that about $10 \%$ of all treated hypertensives on three or more drugs had uncontrolled $\mathrm{BP}^{2}$ Thus, with over 80 million treated hypertensives in the US alone, there appeared to be a vast number of people who could benefit from further intervention and a huge market for new drugs or technologies that were proven to be effective.

In this issue of JGIM, Makai and colleagues ${ }^{3}$ present a network meta-analysis that compares the potential new interventions tested for patients who need something more than the three standard BP drugs to achieve BP control. Without delving into the complexities of the methodology, the conclusion that none of the options reviewed was clearly superior to mineralocorticoid receptor antagonists (MRAs)-spironolactone and eplerenone-is reassuring, as, unlike MRAs, none of the other interventions included in the meta-analysis have been proven safe and effective in their own right, and are not available outside of research settings in this country. Furthermore, it is unlikely that we will know enough about their long-term effectiveness and safety to envision them as part of our therapeutic arsenal in the foreseeable future.

Developing new drugs and especially new devices for resistant hypertension has engendered much excitement in the hypertension field since about 2000. Unfortunately, the results to date have been disappointing. New drug development has always been expensive and risky. The success of Gilead Sciences' hepatitis C drug, sofosbuvir, was not replicated with

Published online April 28, 2017 darusentan, the company's drug for resistant hypertension. Darusentan's side effect profile was a bit worrisome in its most successful trial, and it failed to beat placebo for the prespecified primary endpoint of office BP in a larger trial; the sponsor stopped development in 2009 . $^{4}$

Several types of devices for controlling BP-including renal denervation $(\mathrm{RDN})$, carotid baroreceptor modulation, median nerve modulation, deep brain stimulation, and creation of arteriovenous (AV) anastomosis - have been developed and are in various phases of testing. ${ }^{5}$ The most widely used and evaluated are the renal denervation devices. Although many promising studies have been reported around the world, they were not blinded. The field experienced a significant setback when the highly anticipated results from the sham-controlled Symplicity 3 trial were negative. The evidence-based observer is likely to have reached the same conclusions as those recently reported in a Cochrane review of renal denervation: 1) based on low-quality evidence, renal denervation has no effect on major cardiovascular events or renal function; 2) moderatequality evidence shows no effect on BP; and 3) based on lowquality evidence, renal denervation caused an increase in bradycardia episodes. ${ }^{6}$ Nevertheless, much was learned from the early trials, and new trials are underway with better devices and study designs to address some of the challenges in patient selection, adherence monitoring, and BP measurement.

The ROX Coupler- the AV device that was shown in the network meta-analysis to be superior to MRAs for 24-h diastolic blood pressure (DBP) control - has been tested in one unblinded trial of 88 patients, 44 of whom received the device. $^{7}$ The ROX Coupler was initially designed to improve oxygenation in patients with chronic obstructive pulmonary disease (COPD), but was also noted to reduce BP. The device was inserted in a femoral vein, and pushed through to create an openable and closable fistula between the iliac vein and artery. The follow-up time in the trial was 6 months. The impressive drop in systolic blood pressure (SBP) reported in the single unblinded trial was accompanied by a $29 \%$ incidence of venous stenosis, which was treated with venous stenting procedures. The multi-year risks and benefits of this novel therapy are unknown, and further trials are ongoing.

Luckily, although it might not have been clear over a decade ago when the newer interventions began testing, there is now little doubt in the hypertension community as to the next line of therapy in most cases for resistant hypertension after three standard drugs. In this role, MRAs have been shown to be superior to 
other BP drugs as the fourth drug in randomized controlled trials. ${ }^{8,}{ }^{9}$ The MRA was found to reduce SBP an average of $7.4 \mathrm{mmHg}$ versus active comparators such as beta-blockers, improving BP in almost all patients, and bringing over half of patients into full control. Most of the evidence regarding MRAs as a class is with spironolactone, usually at doses of $25-50 \mathrm{mg}$. Spironolactone is a "four-dollar" drug in many pharmacies. Its feared side effect, gynecomastia, occurs in about $5 \%$ of men who take it for resistant hypertension. Eplerenone, which is also generic, does not cause gynecomastia, but it is more expensive and less potent than spironolactone. Although the package insert limits the maximum dose to $100 \mathrm{mg}$ per day, recent work suggests that to obtain the equivalent effect, eplerenone has to be dosed at 4.5 times the dose of spironolactone. ${ }^{10}$ As most patients will also be on an angiotensin-converting enzyme (ACE) inhibitor or angiotensin receptor blocker (ARB), potassium does need to be monitored. Despite the strong evidence to support the efficacy of MRAs, national databases suggest that less than $6 \%$ of hypertensives on four drugs are on an MRA. ${ }^{11}$ This may be another example of very slow diffusion of evidencebased interventions to medical practitioners in the community, especially when generic drugs are not heavily promoted - something that teachers of primary care try to correct.

In interpreting the findings of the network meta-analysis by Makai et al., we must not lose sight of other important considerations in addressing resistant hypertension. The definition of resistant hypertension includes 1) treatment with three or more adequately dosed antihypertensives, 2) confirmation that the patient is taking the medications as described, and 3) documentation that the BP is uncontrolled outside the office. As resistant hypertension has received increasing attention from the research community and industry groups, it has become clear that it is not always easy to establish whether all three criteria have been met. To solve this problem, the term "apparent treatment-resistant hypertension" has gained currency. The number of resistant hypertensives who truly need more effective therapies than are currently available is likely a function of how much effort is made to identify them. In one US sample, of those who appeared to be resistant on the basis of office BP and prescribed regimen, 29\% had controlled BP on 24-h monitoring and $29 \%$ were non-adherent based on electronic bottle cap monitoring. In this study, as in others that describe resistant hypertension samples, even when patients are on a three-drug regimen that includes a diuretic, the combination of drugs and dosing is frequently suboptimal. Diuretics are the principal tool for reducing difficult-to-control BP. Many experts believe that chlorthalidone should be the preferred diuretic, but it is rarely used in practice. Hydrochlorothiazide is almost never used at doses greater than $25 \mathrm{mg}$ per day. ${ }^{11}$ This dose is much less potent than chlorthalidone $25 \mathrm{mg}$ and may not be adequate for resistant hypertension. One large Israeli study with good medication and refill records found that only $0.8 \%$ of hypertensives who were $85 \%$ adherent to three medications, including a diuretic, had uncontrolled BP. ${ }^{12}$
The most useful technological innovations for managing resistant hypertension may be in the areas of adherence promotion and monitoring and in more accurate $\mathrm{BP}$ measurement. As mentioned earlier, it is reassuring that in the general hypertension population, treatment non-adherence is not the overwhelming problem. However, in populations with resistant hypertension referred to hypertension specialty centers or for device intervention, this is not the case. There have been several recent studies in these populations that used serum or urine drug levels to ascertain adherence. These studies have shown very high levels of complete or partial non-adherence. ${ }^{13}$ Interventions in this very small but high-risk population will likely need to be multifactorial, including behavioral approaches. The growing appreciation of the importance of out-of-office BP measurement will help with accurate classification of resistant hypertension. If we adopt new technologies and protocols to move away from the problematic officebased BP measurements that are now the norm, we could also improve the recognition and management of resistant hypertension.

We do not know precisely how many "real' resistant hypertensives would remain after accounting for adherence and outof-office BP, assuming widespread use of chlorthalidone, or even requiring that four drugs including an MRA be included in the new definition of resistant hypertension. Even if it is $1 \%$, given 80 million-plus hypertensives in the US, and over one billion worldwide, there is still a potential need for new drugs or devices. With further development and testing, device therapy could conceivably be safe and inexpensive enough to provide a lifestyle choice similar to LASIK eye surgery instead of glasses or contacts. There may be a few patients with very high-risk hypertension who are not amenable to improved adherence, in whom device therapy could be lifesaving. The immediate challenge is for primary care physicians and health systems to use the existing drug arsenal, along with behavioral interventions and BP measurement technologies, to further reduce uncontrolled BP. If the already available tools were properly deployed, it is likely that BP control in the US population could reach $98 \%$.

Corresponding Author: David J. Hyman, MD, MPH; Baylor College of Medicine, Houston, TX, USA (e-mail: dhyman@bcm.edu).

\section{Compliance with ethical standards:}

Conflict of Interest: The authors have no confilicts of interest.

\section{REFERENCES}

1. Rose AJ, Berlowitz DR, Orner MB, Kressin NR. Understanding uncontrolled hypertension: is it the patient or the provider? $\mathrm{J}$ Clin Hypertens 2007;9(12):937-943.

2. Judd E, Calhoun DA. Management of Resistant Hypertension: Do Not Give Up on Medication. Nephrol Self Assess Program 2014;13(2):57-63. 
3. Makai P. A network meta-analysis of clinical management strategies for treatment- resistant hypertension: making optimal use of the evidence. $\mathrm{J}$ Gen Intern Med. doi:10.1007/s11606-017-4000-7.

4. Enseleit F, Ruschitzka F. Darusentan in resistant hypertension: lost in translation. Curr Hypertens Rep 2010;12(1):1-3.

5. Ng FL, Saxena M, Mahfoud F, Pathak A, Lobo MD. Device-based Therapy for Hypertension. Curr Hypertens Rep 2016;18(8):61.

6. Coppolino G, Pisano A, Rivoli L, Bolignano D. Renal denervation for resistant hypertension. Cochrane Database Syst Rev 2017;2:CD011499.

7. Lobo MD, Sobotka PA, Stanton A, et al. Central arteriovenous anastomosis for the treatment of patients with uncontrolled hypertension (the ROX CONTROL HTN study): a randomised controlled trial. Lancet 2015;385(9978):1634-1641.

8. Sinnott SJ, Tomlinson LA, Root AA, et al. Comparative effectiveness of fourth-line anti-hypertensive agents in resistant hypertension: A systematic review and meta-analysis. Eur J Prev Cardiol 2017;24(3):228-238.
9. Dudenbostel T, Ghazi L, Liu M, Li P, Oparil S, Calhoun DA. Body Mass Index Predicts 24-Hour Urinary Aldosterone Levels in Patients With Resistant Hypertension. Hypertension 2016;68(4):995-1003.

10. Roush GC, Ernst ME, Kostis JB, Yeasmin S, Sica DA. Dose doubling, relative potency, and dose equivalence of potassium-sparing diuretics affecting blood pressure and serum potassium: systematic review and meta-analyses. J Hypertens 2016;34(1):11-19.

11. Hanselin MR, Saseen JJ, Allen RR, Marrs JC, Nair KV. Description of antihypertensive use in patients with resistant hypertension prescribed four or more agents. Hypertension 2011;58(6):1008-1013.

12. Weitzman D, Chodick G, Shalev V, Grossman C, Grossman E. Prevalence and factors associated with resistant hypertension in a large health maintenance organization in Israel. Hypertension 2014;64(3):501507.

13. Hyman DJ, Pavlik V. Medication adherence and resistant hypertension. J Hum Hypertens 2015;29(4):213-218. 